\title{
New Absolute Stability Conditions of Lur'e Systems with Time-Varying Delay
}

\author{
Wei Wang ${ }^{1}$ and Hong-Bing Zeng ${ }^{2}$ \\ ${ }^{1}$ Hunan Railway Professional Technology College, Zhuzhou 412001, China \\ ${ }^{2}$ School of Electrical and Information Engineering, Hunan University of Technology, Zhuzhou 412008, China \\ Correspondence should be addressed to Hong-Bing Zeng; 9804zhb@163.com
}

Received 13 August 2014; Accepted 18 February 2015

Academic Editor: Petko Petkov

Copyright (C) 2015 W. Wang and H.-B. Zeng. This is an open access article distributed under the Creative Commons Attribution License, which permits unrestricted use, distribution, and reproduction in any medium, provided the original work is properly cited.

This paper is focused on the absolute stability of Lure systems with time-varying delay. Based on the quadratic separation framework, a complete delay-decomposing Lyapunov-Krasovskii functional is constructed. By considering the relationship between the time-varying delay and its varying interval, improved delay-dependent absolute stability conditions in terms of linear matrix inequalities (LMIs) are obtained. Moreover, the derived conditions are extended to systems with time-varying structured uncertainties. Finally, a numerical example is given to show the advantage over existing literatures.

\section{Introduction}

Since the concept of absolute stability and the Lur'e problem was introduced in 1940s, the absolute stability of Lur'e control systems has received much attention and many rich results have been proposed [1-6]. As time-delay is frequently encountered in practical systems and is often a source of instability and poor performance, the problem of absolute stability of Lure systems with time-delay has been attracting much attention [7-14].

In $[10,11]$, the absolute stability of Lure systems with time-invariant delay was addressed. In the case that the delay is time-varying, the problem of absolute stability has also been investigated in [12]. In addition, by retaining some useful information and employing an improved free-matrix approach to consider the relationship between the timevarying delay and its upper bound, some less conservative criteria are obtained in [13]. Nevertheless, the results obtained in [13] are based on simple Lyapunov-Krasovskii functionals and are still conservative. In [14], the absolute stability of Lur'e systems with interval-varying delay has been investigated via a delay decomposition approach. However, there is room for further improvement.

Recently, a delay decomposition method was proposed in [15], which significantly reduced the conservatism of the derived stability conditions for systems with time-varying delay. However, as pointed out in [16], the term $\tau(t)-(k-1) \delta$ with $(k-1) \delta \leq \tau(t) \leq k \delta$ was enlarged as $\delta$ and another term $k \delta-\tau(t)$ was also regarded as $\delta$ in [15], leading to the conservatism. Moreover, in the construction of LyapunovKrasovskii functional, the term $\int_{t-\tau(t)}^{t} x^{T}(s) Q x(s) d s$ has been universally employed in [15]; that is, $Q \geq 0$ is kept on the whole delay interval $[t-\tau(t), t]$, which is also a source of conservatism for systems with time-varying delay.

In this paper, a complete-decomposing LyapunovKrasovskii functional is employed to investigate the absolute stability of Lure systems with a time-varying delay. By considering the relationship between the time-varying delay and its varying interval, improved delay-dependent absolute stability conditions are presented in the linear matrix inequality (LMI) setting. Finally, a numerical example is given to demonstrate the effectiveness and the merits of the presented method.

Notation. Throughout this paper, the superscripts "-1" and " $T$ " stand for the inverse and transpose of a matrix, respectively; $R^{n}$ denotes the $n$-dimensional Euclidean space; $R^{n \times m}$ is the set of all $n \times m$ real matrices; $P>0$ means that the matrix $P$ is symmetric and positive definite; $I$ is an appropriately 
dimensioned identity matrix; $\operatorname{diag}\{\cdots\}$ denotes a blockdiagonal matrix; and the symmetric terms in a symmetric matrix are denoted by $*$; for example, $\left[\begin{array}{ll}X & Y \\ * & Z\end{array}\right]=\left[\begin{array}{cc}X & Y \\ Y^{T} & Z\end{array}\right]$.

\section{Problem Statement}

Consider the following system:

$$
\begin{gathered}
\dot{x}(t)=A x(t)+B x(t-d(t))+D w(t) \\
z(t)=M x(t)+N x(t-d(t)) \\
w(t)=-\varphi(t, z(t)) \\
x(t)=\psi(t), \quad t \in[-h, 0],
\end{gathered}
$$

where $x(t) \in R^{n}, w(t) \in R^{m}$, and $z(t) \in R^{m}$ are the state, input, and output vectors of the system, respectively; $A, B, D, M$, and $N$ are constant matrices with appropriate dimensions; the initial condition $\psi(t)$ is a continuous vectorvalued function of $t \in[-h, 0] . \varphi(t, z(t)) \in R^{m}$ is a nonlinear function, which is piecewise continuous in $t$, globally Lipschitz in $z(t), \varphi(t, 0)=0$, and satisfies the following sector condition $\forall t \geq 0$ and $\forall z(t) \in R^{m}$ :

$$
\left[\varphi(t, z(t))-K_{1} z(t)\right]^{T}\left[\varphi(t, z(t))-K_{2} z(t)\right] \leq 0,
$$

where $K_{1}$ and $K_{2}$ are constant real matrices of appropriate dimensions, and $\bar{K}=K_{2}-K_{1}$ is a symmetric positive definite matrix. Customarily, the nonlinear function, $\varphi(t, z(t))$, is said to belong to the sector $\left[K_{1}, K_{2}\right]$.

The time-delay, $d(t)$, is a time-varying differentiable function satisfying

$$
\begin{gathered}
0 \leq d(t) \leq h, \\
\dot{d}(t) \leq \mu,
\end{gathered}
$$

where $h$ and $\mu$ are constants.

First, we introduce the following definition of absolute stability.

Definition 1. System (1) is said to be absolutely stable in the sector $\left[K_{1}, K_{2}\right]$ if a trivial solution $x(t)=0$ is globally uniformly asymptotically stable for any nonlinear function $\varphi(t, z(t))$ satisfying (2).

In this paper, we investigate not only the absolute stability of nominal system (1), but also the following system with time-varying structured uncertainties:

$$
\begin{gathered}
\dot{x}(t)=(A+\Delta A(t)) x(t) \\
+(B+\Delta B(t)) x(t-d(t))+D w(t) \\
z(t)=M x(t)+N x(t-d(t)) \\
w(t)=-\varphi(t, z(t)) \\
x(t)=\psi(t), \quad t \in[-h, 0],
\end{gathered}
$$

where the time-varying structured uncertainties are of the form

$$
[\Delta A(t) \Delta B(t)]=L F(t)\left[E_{a} E_{b}\right]
$$

$L, E_{a}$, and $E_{b}$ are appropriately dimensioned constant matrices, and $F(t)$ is an unknown real and possibly time-varying matrix satisfying

$$
F^{T}(t) F(t) \leq I, \quad \forall t
$$

Before presenting our main results, we first introduce two lemmas, which are useful in the stability analysis of the considered system.

Lemma 2 (see [10]). Let $M=M^{T}>0$ be a constant real $n \times n$ matrix, and suppose $\dot{x}:[-h, 0] \mapsto R^{n}$ with $h>0$ such that the subsequent integration is well defined. Then, we have

$$
-h \int_{t-h}^{t} \dot{x}^{T}(s) M \dot{x}(s) d s \leq \zeta^{T}(t)\left[\begin{array}{cc}
-M & M \\
* & -M
\end{array}\right] \zeta(t),
$$

where $\zeta(t)=\operatorname{col}\{x(t), x(t-h)\}$

Lemma 3 (see [17]). Let $H, E$, and $F(t)$ be real matrices of appropriate dimensions with $F(t)$ satisfying $F^{T}(t) F(t) \leq I$. Then, for any scalar $\varepsilon>0$

$$
H F(t) E+(H F(t) E)^{T} \leq \varepsilon^{-1} H H^{T}+\varepsilon E^{T} E .
$$

\section{Main Results}

Firstly, by applying the loop transformation the absolute stability of system (1) in the sector $\left[K_{1}, K_{2}\right]$ can be converted to that of the following system:

$$
\begin{gathered}
\dot{x}(t)=\left(A-D K_{1} M\right) x(t) \\
+\left(B-D K_{1} N\right) x(t-d(t))+D w(t) \\
z(t)=M x(t)+N x(t-d(t)) \\
w(t)=-\varphi(t, z(t)) \\
x(t)=\psi(t), \quad t \in[-h, 0]
\end{gathered}
$$

in the sector $\left[0, K_{2}-K_{1}\right]$. Thus, we consider the absolute stability of system (10) in the sector $\left[0, K_{2}-K_{1}\right][18]$.

Now, we decompose the delay interval $[0, h]$ into $m$ equidistant subintervals, where $m$ is a given integer; that is, $[0, h]=\bigcup_{j=1}^{m}[(j-1) \delta, j \delta]$ with $\delta=h / m$. Thus, for any $t \geq 0$, there should exist an integer $k \in\{1,2, \ldots, m\}$, such that $d(t) \in[(k-1) \delta, k \delta]$. Then the Lyapunov-Krasovskii functional candidate is chosen as

$$
\left.V\left(x_{t}\right)\right|_{d(t) \in[(k-1) \delta, k \delta]}=\sum_{j=1}^{4} V_{j}\left(x_{t}\right) \text {, }
$$


where

$$
\begin{gathered}
V_{1}\left(x_{t}\right)=x^{T}(t) P x(t) \\
V_{2}\left(x_{t}\right)=\int_{t-\delta}^{t} \zeta_{1}^{T}(s) R_{a} \zeta_{1}(s) d s \\
V_{3}\left(x_{t}\right)=\sum_{j=1}^{m} \delta \int_{-j \delta}^{-(j-1) \delta} \int_{t+\theta}^{t} \dot{z}^{T}(s) Z_{j} \dot{z}(s) d s d \theta \\
V_{4}\left(x_{t}\right)=\sum_{j=1}^{k-1} \int_{t-j \delta}^{t-(j-1) \delta} x^{T}(s) Q_{j} x_{2}(s) d s \\
+\int_{t-d(t)}^{t-(k-1) \delta} x^{T}(s) Q_{k} x(s) d s
\end{gathered}
$$

where $P>0, R_{a}=\left[\begin{array}{cccc}R_{11} & R_{12} & \cdots & R_{1 m} \\ * & R_{22} & \cdots & R_{2 m} \\ * & * & \ddots & \vdots \\ * & * & * & R_{m m}\end{array}\right]>0, Q_{j} \geq 0$, and $Z_{j}>0, j=1,2, \ldots, m$, are to be determined, and $\zeta_{1}(s)=$ $\left[\begin{array}{llll}x^{T}(s) & x^{T}(s-\delta) & \cdots & x^{T}(s-(m-1) \delta)\end{array}\right]^{T}$.

Remark 4. Recently, the delay-decomposing approach is employed to reduce the conservatism of stability analysis for systems with time-varying delay in [15]. However, the technique proposed in this paper improves the existing ones. In Lyapunov-Krasovskii functional (11), the quadratic separation is employed in $V_{2}\left(x_{t}\right)$, and a new term, $V_{4}\left(x_{t}\right)$, is introduced, which play an important role in reducing conservatism of the resulting conditions.

For the absolute stability of system (1), we have the following result.

Theorem 5. Given a positive integer $m$ and scalars $h>0$ and $\mu$, system (1) with a time-varying delay $d(t)$ satisfying (3) and (4) is absolutely stable in the sector $\left[K_{1}, K_{2}\right]$ if there exist matrices $P>0, R_{a}=\left[\begin{array}{cccc}R_{11} & R_{12} & \cdots & R_{1 m} \\ * & R_{22} & \cdots & R_{2 m} \\ * & * & \ddots & \vdots \\ * & * & * & R_{m m}\end{array}\right]>0, Q_{j} \geq 0$, and $Z_{j}>0, j=1,2, \ldots, m$, and any matrices $G_{i j}, H_{i j}, i=1,2$, $j=1,2, \ldots, m$, such that for $k=1,2, \ldots, m$,

$$
\begin{gathered}
{\left[\begin{array}{cc}
\Phi_{0}+\bar{\Phi}_{k}+\Lambda_{k} & \delta \Gamma^{T} \widehat{Z} \\
* & -\widehat{Z}
\end{array}\right]<0} \\
{\left[\begin{array}{cc}
\Phi_{0}+\bar{\Phi}_{k}+\Lambda_{k} & \delta \Gamma^{T} \widehat{Z} \\
* & -\widehat{Z}
\end{array}\right]<0,}
\end{gathered}
$$

where

$$
\begin{aligned}
& \Phi_{0}=\left[\begin{array}{cccccc}
0 & \bar{B}^{T} P & 0 & \cdots & -N^{T} \bar{K}^{T} & 0 \\
* & \varphi_{11} & \varphi_{12} & \cdots & \varphi_{1(m+2)} & 0 \\
* & * & \varphi_{22} & \cdots & \varphi_{2(m+2)} & 0 \\
* & * & * & \ddots & \vdots & \vdots \\
* & * & * & \cdots & \varphi_{(m+2)(m+2)} & 0 \\
* & * & * & \cdots & * & 0
\end{array}\right] \\
& \varphi_{i j}= \begin{cases}\varphi_{1 j}, & i=1,1 \leq j \leq m+2 \\
\varphi_{i(m+1)}, & 2 \leq i \leq m+1, i=m+1 \\
\varphi_{i(m+2)}, & 2 \leq i \leq m+2, i=m+2 \\
\bar{R}_{i j}-\bar{Z}_{i}, & i=j=2,3, \ldots, m \\
\bar{R}_{i j}+Z_{i}, & 2 \leq i \leq m-1, j=i+1 \\
\bar{R}_{i j}, & \text { otherwise }\end{cases} \\
& \varphi_{1 j}= \begin{cases}P \bar{A}+\bar{A}^{T} P+R_{11}-Z_{1}, & j=1 \\
R_{12}+Z_{1}, & j=2 \\
R_{1 j}, & 3 \leq j \leq m \\
0, & j=m+1 \\
P D-M^{T} \bar{K}^{T}, & j=m+2\end{cases} \\
& \varphi_{i(m+1)}= \begin{cases}-R_{(i-1) m}, & 2 \leq i \leq m-1 \\
-R_{(m-1) m}+Z_{m}, & i=m \\
-R_{m m}-Z_{m}, & i=m+1\end{cases} \\
& \varphi_{i(m+2)}= \begin{cases}0, & 2 \leq i \leq m+1 \\
-2 I, & i=m+2\end{cases} \\
& \bar{\Phi}_{k}=\left(\bar{\psi}_{i j}\right)_{(m+4) \times(m+4)}+\left(\bar{\psi}_{i j}\right)_{(m+4) \times(m+4)}^{T} \\
& \bar{\psi}_{i j}= \begin{cases}G_{1 k}-H_{1 k}, & i=j=1 \\
H_{1 k}-H_{2 k}^{T}+G_{2 k}^{T}, & i=1, j=k+1 \\
-G_{1 k}, & i=1, j=k+2 \\
H_{2 k}+\frac{1}{2} Z_{k}, & i=j=k+1 \\
-G_{2 k}-Z_{k}, & i=k+1, j=k+2 \\
\frac{1}{2} Z_{k}, & i=j=k+2 \\
-\delta_{k} H_{1 k}, & i=1, j=m+4 \\
-\delta_{k} H_{2 k}, & i=k+1, j=m+4 \\
-\frac{1}{2} \delta_{k}^{2} Z_{k}, & i=j=m+4 \\
0, & \text { otherwise }\end{cases}
\end{aligned}
$$




$$
\begin{aligned}
& \widehat{\Phi}_{k}=\left(\widehat{\psi}_{i j}^{(2)}\right)_{(m+4) \times(m+4)}+\left(\widehat{\psi}_{i j}\right)_{(m+4) \times(m+4)}^{T} \\
& \widehat{\psi}_{i j}= \begin{cases}G_{1 k}-H_{1 k}, & i=j=1 \\
H_{1 k}-H_{2 k}^{T}+G_{2 k}^{T}, & i=1, j=k+1 \\
-G_{1 k}, & i=1, j=k+2 \\
H_{2 k}+\frac{1}{2} Z_{k}, & i=j=k+1 \\
-G_{2 k}-Z_{k}, & i=k+1, j=k+2 \\
\frac{1}{2} Z_{k}, & i=j=k+2 \\
-\delta_{k} G_{1 k}, & i=1, j=m+4 \\
-\delta_{k} G_{2 k}, & i=k+1, j=m+4 \\
-\frac{1}{2} \delta_{k}^{2} Z_{k}, & i=j=m+4 \\
0, & \text { otherwise }\end{cases} \\
& \Lambda_{k}=\operatorname{diag}\left\{\Lambda_{1 k}, \Lambda_{2 k}, \ldots, \Lambda_{(m+4) k}\right\} \\
& \Lambda_{j k}= \begin{cases}-(1-\mu) Q_{k}, & j=1 \\
Q_{1}, & j=2 \\
Q_{j-1}-Q_{j-2}, & 3 \leq j \leq k+1 \\
0, & \text { otherwise }\end{cases} \\
& \bar{A}=A-D K_{1} M \\
& \bar{B}=B-D K_{1} N \\
& \bar{R}_{i j}=R_{i j}-R_{(i-1)(j-1)} \\
& \bar{Z}_{j}=Z_{j}+Z_{j-1} \\
& \bar{K}=K_{2}-K_{1} \\
& \widehat{Z}=\sum_{j=1}^{m} Z_{j} \\
& \Gamma=\left[\begin{array}{lllllll}
\bar{B} & \bar{A} & 0 & \cdots & 0 & D & 0
\end{array}\right] .
\end{aligned}
$$

Proof. From the Leibniz-Newton formula, the following equations are true for any matrices $G_{i k}, H_{i k}, i=1,2, k=$ $1,2, \ldots, m$ with appropriate dimensions

$$
\begin{aligned}
0= & 2\left[x^{T}(t-d(t)) H_{1 k}+x^{T}(t-(k-1) \delta) H_{2 k}\right] \\
& \times\left[x(t-(k-1) \delta)-x(t-d(t))-\int_{t-d(t)}^{t-(k-1) \delta} \dot{x}(s) d s\right] \\
0= & 2\left[x^{T}(t-d(t)) G_{1 k}+x^{T}(t-(k-1) \delta) G_{2 k}\right] \\
& \times\left[x(t-d(t))-x(t-k \delta)-\int_{t-k \delta}^{t-d(t)} \dot{x}(s) d s\right] .
\end{aligned}
$$

On the other hand, it follows from (2) and (10) that

$$
\begin{array}{r}
-2 w^{T}(t) w(t)-2 w^{T}(t)\left(K_{2}-K_{1}\right) \\
\cdot[M x(t)+N x(t-d(t))] \geq 0 .
\end{array}
$$

Taking the derivative of $V\left(x_{t}\right)$ in (11) with respect to $t$ along the trajectory of system (10) yields

$$
\left.\dot{V}\left(x_{t}\right)\right|_{d(t) \in[(k-1) \delta, k \delta]}=\sum_{j=1}^{4} \dot{V}_{j}\left(x_{t}\right),
$$

where

$$
\begin{gathered}
\dot{V}_{1}\left(x_{t}\right)=2 x^{T}(t) P \dot{x}(t) \\
\dot{V}_{2}\left(x_{t}\right)=\zeta_{1}^{T}(t) R_{a} \zeta_{1}^{T}(t)-\zeta_{1}^{T}(t-\delta) R_{a} \zeta_{1}^{T}(t-\delta) \\
\dot{V}_{3}\left(x_{t}\right)=\delta^{2} \sum_{j=1}^{m} \dot{x}^{T}(t) Z_{j} \dot{x}(t)-\delta \sum_{j=1}^{m} \int_{t-j \delta}^{t-(j-1) \delta} \dot{x}^{T}(s) Z_{j} \dot{x}(s) d s \\
\dot{V}_{4}\left(x_{t}\right)=\sum_{j=1}^{k-1} x^{T}(t-j \delta)\left(Q_{j+1}-Q_{j}\right) x(t-j \delta) \\
+x^{T}(t) Q_{1} x(t) \\
-(1-\dot{d}(t)) x^{T}(t-d(t)) Q_{k} x(t-d(t)) \\
\leq \sum_{j=1}^{k-1} x^{T}(t-j \delta)\left(Q_{j+1}-Q_{j}\right) x(t-j \delta) \\
+x^{T}(t) Q_{1} x(t) \\
-(1-\mu) x^{T}(t-d(t)) Q_{k} x(t-d(t)) .
\end{gathered}
$$

Applying Lemma 2, we have

$$
\begin{gathered}
-\sum_{j=1}^{m} \delta \int_{t-j \delta}^{t-(j-1) \delta} \dot{x}^{T}(s) Z_{j} \dot{x}(s) d s \\
\leq \sum_{j=1, j \neq k}^{m}\left[\begin{array}{c}
x(t-(j-1) \delta) \\
x(t-j \delta)
\end{array}\right]^{T}\left[\begin{array}{cc}
-Z_{j} & Z_{j} \\
* & -Z_{j}
\end{array}\right] \\
\cdot\left[\begin{array}{c}
x(t-(j-1) \delta) \\
x(t-j \delta)
\end{array}\right] \\
-\delta \int_{t-k \delta}^{t-(k-1) \delta} \dot{x}^{T}(s) Z_{k} \dot{x}(s) d s
\end{gathered}
$$




$$
\begin{aligned}
= & \sum_{j=1}^{m}\left[\begin{array}{c}
x(t-(j-1) \delta) \\
x(t-j \delta)
\end{array}\right]^{T}\left[\begin{array}{cc}
-Z_{j} & Z_{j} \\
* & -Z_{j}
\end{array}\right]\left[\begin{array}{c}
x(t-(j-1) \delta) \\
x(t-j \delta)
\end{array}\right] \\
& -\left[\begin{array}{c}
x(t-(j-1) \delta) \\
x(t-j \delta)
\end{array}\right]^{T}\left[\begin{array}{cc}
-Z_{k} & Z_{k} \\
* & -Z_{k}
\end{array}\right]\left[\begin{array}{c}
x(t-(j-1) \delta) \\
x(t-j \delta)
\end{array}\right] \\
& -\delta \int_{t-d(t)}^{t-(k-1) \delta} \dot{x}^{T}(s) Z_{k} \dot{x}(s) d s-\delta \int_{t-k \delta}^{t-d(t)} \dot{x}^{T}(s) Z_{k} \dot{x}(s) d s .
\end{aligned}
$$

Introducing a new vector as

$$
\zeta(t, s)=\left[\begin{array}{c}
x(t-d(t)) \\
x(t) \\
x(t-\delta) \\
x(t-2 \delta) \\
\vdots \\
x(t-m \delta) \\
w(t) \\
\dot{x}(s)
\end{array}\right]
$$

then system (10) gives

$$
\dot{x}(t)=\Gamma \zeta(t, s) .
$$

Adding the right sides of ((16)-(17)) to (18) and applying (20) and (22), we have

$$
\begin{aligned}
& \left.\dot{V}\left(x_{t}\right)\right|_{d(t) \in[(k-1) \delta, k \delta]} \\
& \leq \frac{1}{\delta} \int_{t-d(t)}^{t-(k-1) \delta} \zeta^{T}(t, s) \\
& \cdot\left[\Phi_{0}+\bar{\Phi}_{k}+\Lambda_{k}+\delta^{2} \Gamma^{T} \sum_{j=1}^{m} Z_{j} \Gamma\right] \zeta(t, s) d s \\
& +\frac{1}{\delta} \int_{t-k \delta}^{t-d(t)} \zeta^{T}(t, s) \\
& \cdot\left[\Phi_{0}+\widehat{\Phi}_{k}+\Lambda_{k}+\delta^{2} \Gamma^{T} \sum_{j=1}^{m} Z_{j} \Gamma\right] \zeta(t, s) d s .
\end{aligned}
$$

For all $k=1,2, \ldots, m$, if $\Phi_{0}+\bar{\Phi}_{k}+\Lambda_{k}+\delta^{2} \Gamma^{T} \sum_{j=1}^{m} Z_{j} \Gamma<0$ and $\Phi_{0}+\widehat{\Phi}_{k}+\Lambda_{k}+\delta^{2} \Gamma^{T} \sum_{j=1}^{m} Z_{j} \Gamma<0$, which are equivalent to LMIs (13) and (14) in the sense of Schur complement [19], then $\dot{V}\left(x_{t}\right)<0$ for any $\zeta(t, s) \neq 0$. Noted that $V\left(x_{t}\right)$ is continuous at $d(t)=k \delta$, so the absolute stability of system (10) in the sector $\left[0, K_{2}-K_{1}\right]$, which is equivalent to that of system (1) in the sector $\left[K_{1}, K_{2}\right]$, is achieved. This completes the proof.

For system (5) with time-varying structured uncertainties (6), replacing $A$ and $B$ in (13) and (14) with $A+L F(t) E_{a}$ and
$B+L F(t) E_{b}$, respectively, applying Lemma 3 and the Schur complement, the following theorem can be derived.

Theorem 6. Given a positive integer $m$, scalars $h>0, \mu$, system (5) with time-varying structured uncertainties (6) and a timevarying delay $d(t)$ satisfying (3) and (4) is robustly absolutely stable in the sector $\left[K_{1}, K_{2}\right]$ if there exist matrices $P>0, R_{a}=$ $\left[\begin{array}{cccc}R_{11} & R_{12} & \cdots & R_{1 m} \\ * & R_{22} & \cdots & R_{2 m} \\ * & * & \ddots & \vdots \\ * & * & * & R_{m m}\end{array}\right]$ $>0, Q_{j} \geq 0, Z_{j}>0, j=1,2, \ldots, m$, any matrices $G_{i j}, H_{i j}, i=1,2, j=1,2, \ldots, m$, and scalars $\lambda_{i j}>0$, $i=1,2, j=1,2, \ldots, m$, such that for $k=1,2, \ldots, m$,

$$
\begin{gathered}
{\left[\begin{array}{cccc}
\Phi_{0}+\bar{\Phi}_{k}+\Lambda_{k} & \delta \Gamma^{T} \widehat{Z} & \bar{P} L & \lambda_{1 k} \bar{E} \\
* & -\widehat{Z} & \delta \widehat{Z}^{T} L & 0 \\
* & * & -\lambda_{1 k} I & 0 \\
* & * & * & -\lambda_{1 k} I
\end{array}\right]<0} \\
{\left[\begin{array}{cccc}
\Phi_{0}+\widehat{\Phi}_{k}+\Lambda_{k} & \delta \Gamma^{T} \widehat{Z} & \bar{P} L & \lambda_{2 k} \bar{E} \\
* & -\widehat{Z} & \delta \widehat{Z}^{T} L & 0 \\
* & * & -\lambda_{2 k} I & 0 \\
* & * & * & -\lambda_{2 k} I
\end{array}\right]<0,}
\end{gathered}
$$

where

$$
\begin{aligned}
\bar{P} & =\left[\begin{array}{lllll}
0 & P & 0 & \cdots & 0
\end{array}\right]^{T} \\
\bar{E} & =\left[\begin{array}{lllll}
E_{b} & E_{a} & 0 & \cdots & 0
\end{array}\right]^{T}
\end{aligned}
$$

and $\Phi_{0}, \bar{\Phi}_{k}, \widehat{\Phi}_{k}, \Lambda_{k}, \Gamma$, and $\widehat{Z}$ are defined in Theorem 5 .

Remark 7. In previous works such as $[12,13,15]$, considerable attention has been paid to the case that the derivative of the time-varying delay $\dot{d}(t)$ satisfies (4). In the case that $\dot{d}(t)$ have different upper bounds in various delay intervals, that is,

$$
\dot{d}(t) \leq \mu_{k}, \quad d(t) \in[(k-1) \delta, k \delta], k=1,2, \ldots, m,
$$

the treatment in $[12,13]$ means that $\dot{d}(t)$ in (26) is enlarged to $\dot{d}(t) \leq \mu=\max \left\{\mu_{1}, \mu_{2}, \ldots, \mu_{m}\right\}$, which may inevitably lead to conservativeness. Nevertheless, replacing $\mu$ with $\mu_{k}$ in Theorems 5 and 6, some less conservative conditions are readily obtained, in which the aforementioned case can be taken fully into account. In addition, for the case that the time-varying delay $d(t)$ is nondifferentiable in a subinterval $d(t) \in[(k-1) \delta, k \delta], k \in\{1,2, \ldots, m\}$, setting corresponding matrix $Q_{k}=0$ in Theorems 5 and 6, the corresponding criteria can be derived. For the limitation of pages, we omit them.

\section{Numerical Example}

In this section, we will present a numerical example to show the effectiveness of the proposed method. 
TABLE 1: Maximum allowable time-delay bounds $h$ for different $\mu$.

\begin{tabular}{lcccc}
\hline$\mu$ & 0.0 & 0.3 & 0.6 & 0.9 \\
\hline$[12]$ & 3.3057 & 2.0787 & 1.4195 & 0.9228 \\
{$[13]$} & 3.3057 & 2.2262 & 1.7409 & 1.4682 \\
{$[14](m=2)$} & 4.1076 & 2.4136 & 1.7457 & 1.6007 \\
{$[14](m=3)$} & 4.2657 & 2.4700 & 1.8185 & 1.6817 \\
Theorem 6 $(m=2)$ & 4.1076 & 2.4660 & 1.8787 & 1.7190 \\
Theorem 6 $(m=3)$ & 4.2664 & 2.5164 & 1.9147 & 1.7923 \\
\hline
\end{tabular}

Example 1. Consider the robust absolute stability of the uncertain system (5) with the following parameters:

$$
\begin{gathered}
A=\left[\begin{array}{cc}
-2 & 0 \\
0 & -0.9
\end{array}\right], \quad B=\left[\begin{array}{cc}
-1 & 0 \\
-1 & -1
\end{array}\right], \quad D=\left[\begin{array}{r}
-0.2 \\
-0.3
\end{array}\right] \\
M=\left[\begin{array}{ll}
0.3 & 0.1
\end{array}\right], \quad N=\left[\begin{array}{ll}
0.1 & 0.2
\end{array}\right], \quad K_{1}=0.2 \\
K_{2}=0.5, \quad L=\left[\begin{array}{cc}
0.1 & 0 \\
0 & 0.1
\end{array}\right], \quad E_{a}=E_{b}=\left[\begin{array}{ll}
1 & 0 \\
0 & 1
\end{array}\right] .
\end{gathered}
$$

For different $m$ and $\mu$, the maximum allowable time-delay bounds (MATB) obtained by Theorem 6 and those methods in [12-14] are listed in Table 1. It is clear that our results are less conservative than those in [12-14]. Furthermore, it can be concluded that the larger the $m$ is, the less conservative results we obtain and the more computing time we consume. As a compromise between less conservative results and CPU computing time, taking $m=2$ or $m=3$ is a good choice.

\section{Conclusions}

In this paper, the problem of absolute stability of Lur'e systems with a time-varying delay has been investigated. A complete-decomposing Lyapunov-Krasovskii functional has been proposed, in which all integral terms including delay are decomposed. By introducing some free-weighting matrices to consider the relationship between time-varying delay and its varying interval, some improved conditions have been derived. A numerical example has been given to demonstrate the superiority over the existing ones.

\section{Conflict of Interests}

The authors declare that there is no conflict of interests regarding the publication of this paper.

\section{Acknowledgments}

This work was supported by the National Natural Science Foundation of China under Grant nos. 61304064, 61273157, and 61203136 and the National Science Fund for Youth Scholars of Hunan Province under Grant no. 2015JJ3064.

\section{References}

[1] A. I. Lur'e, Some Nonlinear Problems in the Theory of Automatic Control, H.M. Stationery Office, London, UK, 1957.

[2] V.-M. Popov, Hyperstability of Control Systems, Springer, New York, NY, USA, 1973.

[3] C. Yang, Q. Zhang, and L. Zhou, "Generalised absolute stability analysis and synthesis for Lur'e-type descriptor systems," IET Control Theory \& Applications, vol. 1, no. 3, pp. 617-623, 2007.

[4] X. Liao, Z. Chen, F. Xu, and P. Yu, "Robust absolute stability of Lurie interval control systems," International Journal of Robust and Nonlinear Control, vol. 17, no. 18, pp. 1669-1689, 2007.

[5] Z.-G. Wu, P. Shi, H. Su, and J. Chu, "Sampled-data synchronization of chaotic Lur'e systems with time delays," IEEE Transactions on Neural Networks and Learning Systems, vol. 24, no. 3, pp. 410-421, 2013.

[6] C. K. Zhang, L. Jiang, Y. He, Q. H. Wu, and M. Wu, "Asymptotical synchronization for chaotic Lur'e systems using sampled-data control," Communications in Nonlinear Science and Numerical Simulation, vol. 18, no. 10, pp. 2743-2751, 2013.

[7] H.-B. Zeng, Y. He, M. Wu, and S.-P. Xiao, "Absolute stability and stabilization for Lurie networked control systems," International Journal of Robust and Nonlinear Control, vol. 21, no. 14, pp. 16671676, 2011.

[8] Q. L. Han, "A new delay-dependent absolute stability criterion for a class of nonlinear neutral systems," Automatica, vol. 44, no. 1, pp. 272-277, 2008.

[9] X. Liu, Q. Gao, and L. Niu, "A revisit to synchronization of Lurie systems with time-delay feedback control," Nonlinear Dynamics, vol. 59, no. 1-2, pp. 297-307, 2010.

[10] Q.-L. Han, "Absolute stability of time-delay systems with sectorbounded nonlinearity," Automatica, vol. 41, no. 12, pp. 21712176, 2005.

[11] M. Wu, Z.-Y. Feng, and Y. He, "Improved delay-dependent absolute stability of Lur'e systems with time-delay," International Journal of Control, Automation and Systems, vol. 7, no. 6, pp. 1009-1014, 2009.

[12] Q.-L. Han and D. Yue, "Absolute stability of Lur'e cprime systems with time-varying delay," IET Control Theory \& Applications, vol. 1, no. 3, pp. 854-859, 2007.

[13] M. Wu, Z. Y. Feng, Y. He, and J. H. She, "Improved delaydependent absolute stability and robust stability for a class of nonlinear systems with a time-varying delay," International Journal of Robust and Nonlinear Control, vol. 20, no. 6, pp. 694702, 2010.

[14] H.-B. Zeng, Y. He, M. Wu, and S.-P. Xiao, "Further results on absolute stability of Lur'e systems with interval time-varying delay," in Proceedings of the 24th Chinese Control and Decision Conference (CCDC '12), pp. 541-545, IEEE, Taiyuan, China, May 2012.

[15] X.-M. Zhang and Q.-L. Han, "A delay decomposition approach to delay-dependent stability for linear systems with timevarying delays," International Journal of Robust and Nonlinear Control, vol. 19, no. 17, pp. 1922-1930, 2009.

[16] H.-B. Zeng, Y. He, M. Wu, and S.-P. Xiao, "Less conservative results on stability for linear systems with a time-varying delay," Optimal Control Applications \& Methods, vol. 34, no. 6, pp. 670679, 2013.

[17] I. R. Petersen and C. V. Hollot, "A Riccati equation approach to the stabilization of uncertain linear systems," Automatica, vol. 22, no. 4, pp. 397-411, 1986. 
[18] H. K. Khalil, Nonlinear Systems, Prentice Hall, Upper Saddle River, NJ, USA, 1996.

[19] S. Boyd, L. El Ghaoui, E. Feron, and V. Balakrishnan, Linear Matrix Inequalities in System and Control Theory, vol. 15 of SIAM Studies in Applied Mathematics, Society for Industrial and Applied Mathematics (SIAM), Philadelphia, Pa, USA, 1994. 

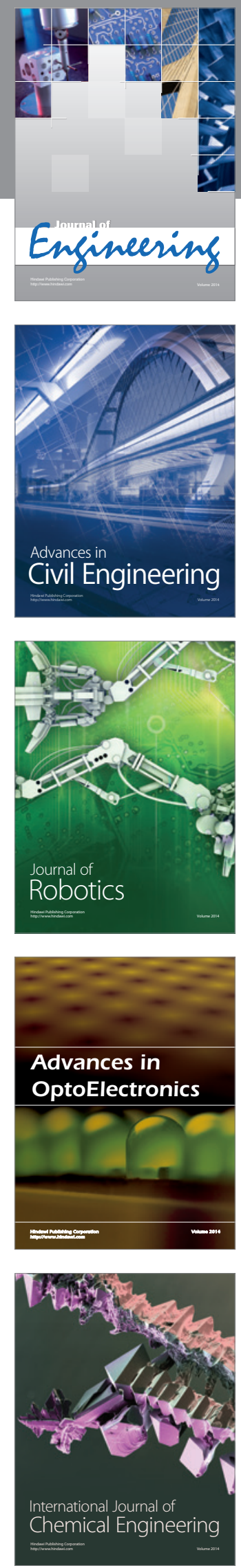

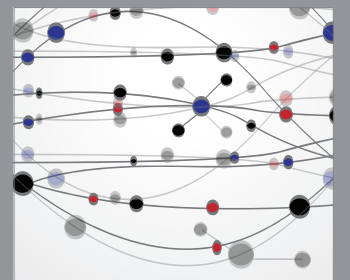

The Scientific World Journal
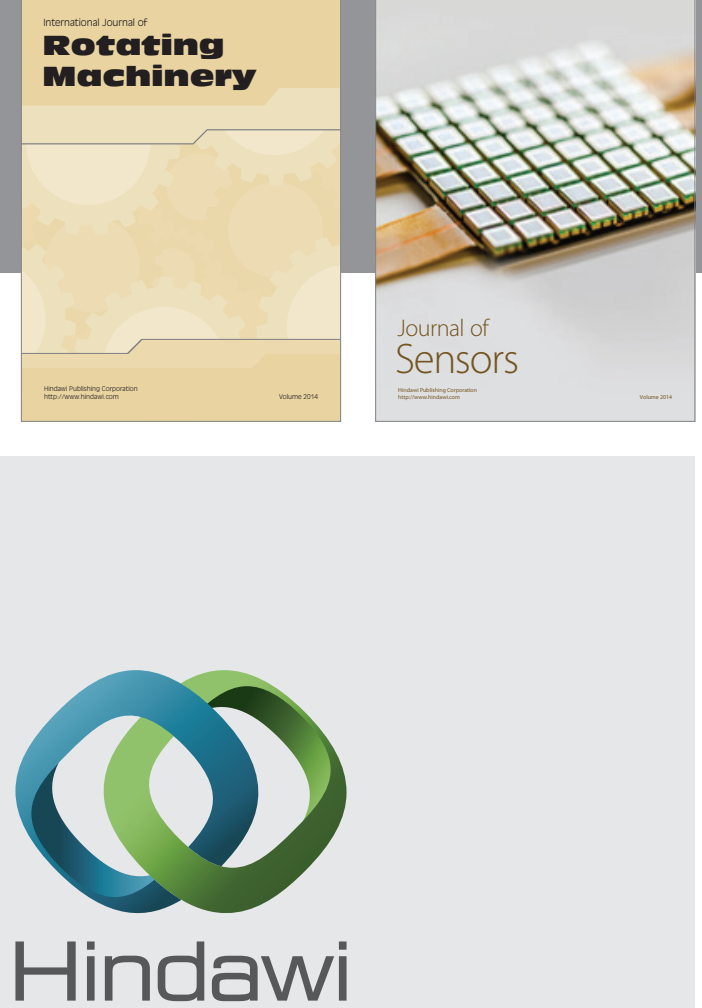

Submit your manuscripts at http://www.hindawi.com
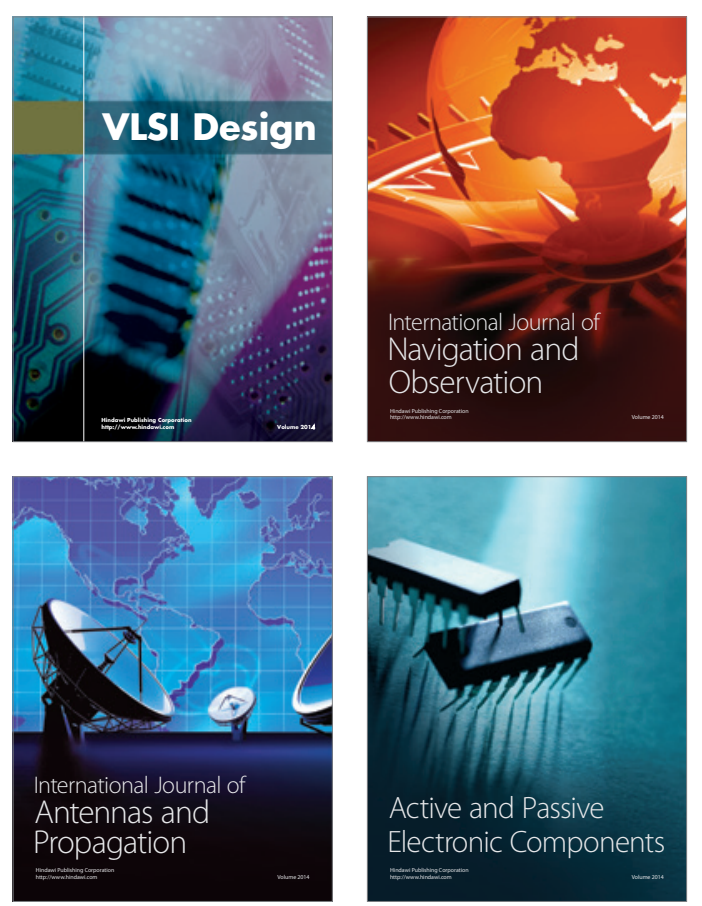
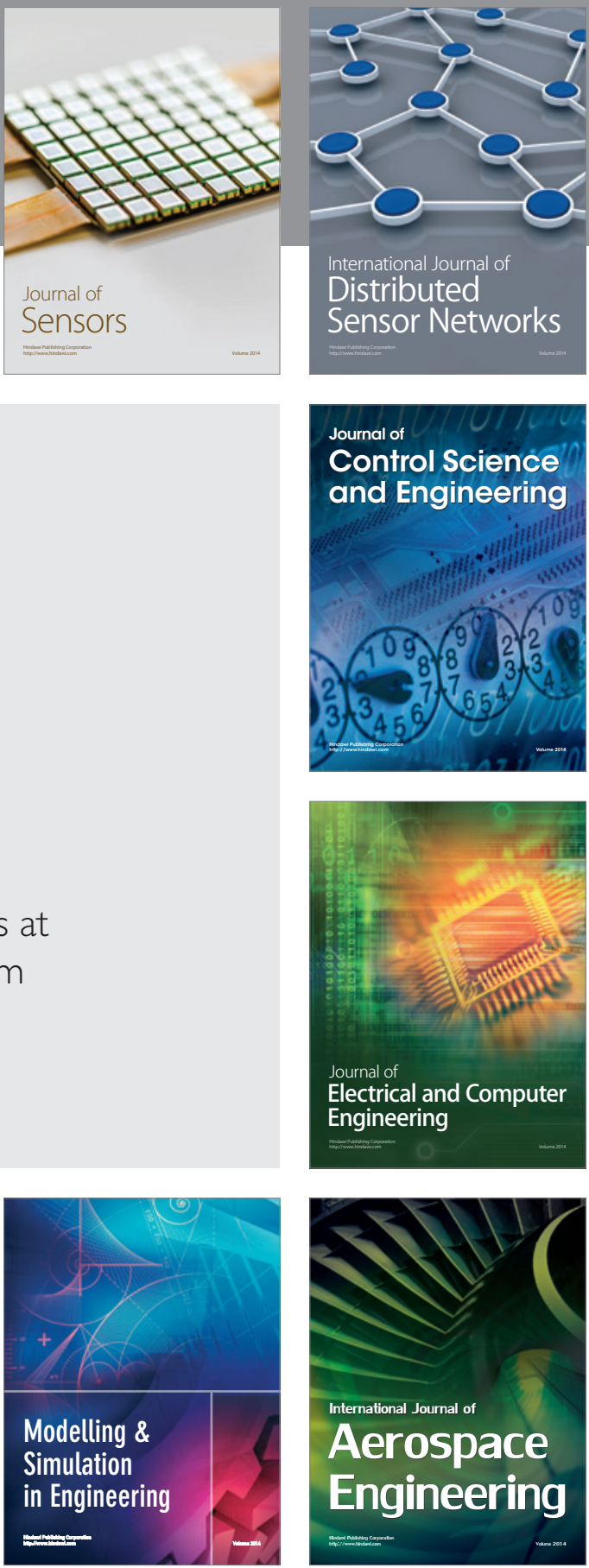

Journal of

Control Science

and Engineering
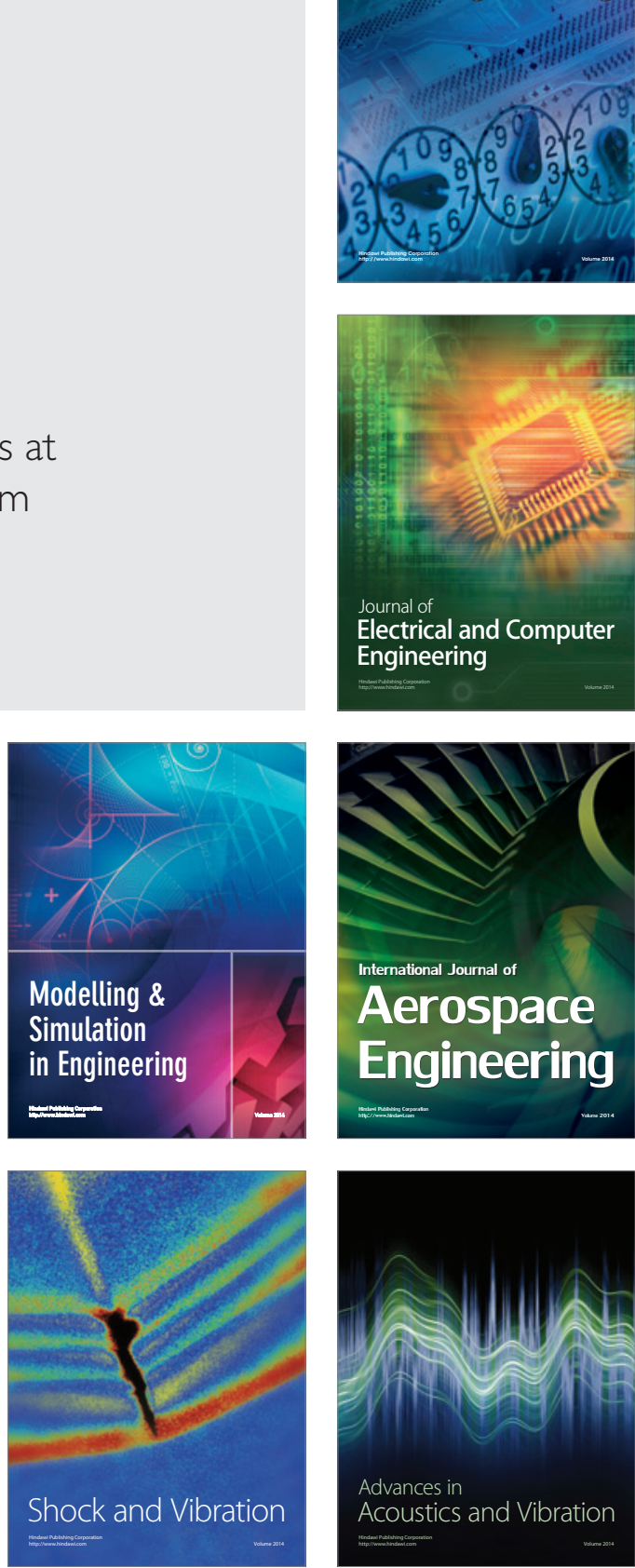